\title{
PROPIEDAD SOBRE LOS FONDOS DE PENSIONES $Y$ CONTINUIDAD DE LAS PRESTACIONES PARA LOS TRABAJADORES TÉCNICOS EXTRANJEROS*
}

¿Los técnicos extranjeros poseen los mismos derechos que el resto de los afiliados sobre sus fondos en las AFPs? Es la pregunta que nos planteamos al analizar esta sentencia de la Corte de Apelaciones de Santiago de 24 de junio de 2013. Una primera aproximación a este tema nos lleva a señalar que los técnicos extranjeros se encuentran en una situación privilegiada. Esto debido a que pueden hacer el retiro de los fondos, tal como sucede en el caso en análisis. Sin embargo, esta situación más favorable a nuestro parecer es sólo aparente.

En el presente comentario analizaremos, en primer término, los hechos que dieron lugar a la sentencia, para luego abocarnos a la explicación de la legislación aplicada a este caso. Finalmente, señalaremos cómo este tipo de reglamentación, aunque se encuentra acorde a las regulaciones internaciones, puede resultar perjudicial para los trabajadores migrantes.

La sentencia en comento resuelve un recurso de protección presentado por don Juan Agustín Arturo, quien presenta esta acción constitucional en contra de AFP Habitat S.A., alegando que dicha AFP no le devolvió los fondos en esta acumulados y que son de su propiedad. Fondos que corresponden a cotizaciones enteradas entre el 26 de noviembre de 1992 y el 11 de mayo de 1994, que equivalen a la suma de \$ 6.052.039 pesos chilenos. Se agrega que luego el actor volvió a Argentina, país en el cual se encontraba afiliado al régimen de seguridad social. El año 2008 el actor intenta recuperar los fondos acumulados, sin embargo, la AFP se niega a esto y le solicita que acompañe una copia

\footnotetext{
* Colaboración recibida el 16 de octubre y aprobada el 25 de octubre de 2013.

Este trabajo forma parte de los proyectos Fondecyt Iniciación № 11121677, "La coordinación de sistemas de seguridad social: Estudio comparado Unión Europea e Iberoamérica" y del Proyecto DID № S-2012-44 Universidad Austral de Chile, "Los mecanismos de coordinación de sistemas de seguridad social: Europa e Iberoamérica".

** Doctor en Derecho de la Université Paris Ouest Nanterre La Defense, Francia. Profesor de Derecho del Trabajo y de Seguridad Social, Universidad Austral de Chile, Valdivia. Dirección postal: Universidad Austral de Chile, Facultad de Ciencias Jurídicas y Sociales, Campus Isla Teja S/N, Valdivia, Chile. Correo electrónico: pablo.arellano@uach.cl.
} 
del contrato de trabajo en Chile. Dicho contrato no se logró acreditar pero sin embargo el actor poseía un finiquito de su relación de trabajo en Chile.

La Corte de Apelaciones fundamentándose, principalmente, en el derecho de propiedad acoge la acción y ordena que se entreguen los dineros al trabajador extranjero en vista que éste cumple con los requisitos establecidos en la Ley $N^{\circ} 18.156$, indicando que la no existencia de contrato de trabajo no puede ser una limitante, cuando existe un finiquito, para que la AFP cumpla con su obligación de entregar los fondos acumulados.

Este caso llama la atención por muchas razones. Por ejemplo, se le adjudica valor probatorio a un finiquito. Además, sorprende que se presente un recurso de protección en contra de una AFP. En contra de una Isapre ya estamos habituados pero no en contra de una AFP.

Nuestra intención en este comentario es centrarnos en la propiedad sobre los fondos acumulados. Este caso y la reglamentación existente hacen notar la existencia de un derecho de propiedad sobre los fondos, distinto al que tienen el común de los afiliados. Los técnicos extranjeros pueden retirar los fondos antes de la edad para jubilar, sin que esto implique que utilizaran dichos dineros para incrementar el monto de su pensión. Analizaremos a continuación cómo regula esta situación nuestra legislación, para luego hacer algunos comentarios basados en la reglamentación internacional protectora de la seguridad social de los trabajadores migrantes.

Nuestra legislación permite que el extranjero que realiza labores en nuestro país no cotice a nuestro sistema bajo ciertas condiciones. En primer lugar, debemos afirmar que la legislación chilena, para la incorporación al sistema de seguridad social no hace diferencia entre un trabajador extranjero y un trabajador nacional, siendo obligatorio cotizar para todo trabajador dependiente. Sin embargo, se establecen exenciones para cierto tipo de trabajadores en disposiciones legales contenidas en Convenios Bilaterales de Seguridad Social y en la Ley $N^{\circ} 18.156$, publicada en el Diario Oficial el 25.08.1982, relativa a Técnicos Extranjeros.

Nos detendremos en la Ley $N^{\circ} 18.156$. Esta establece la exención de efectuar cotizaciones para los trabajadores técnicos extranjeros, como para las empresas que los contraten cumpliendo con los requisitos establecidos en su artículo $1^{\circ}$, a saber:

"Las empresas que celebren contratos de trabajo con personal técnico extranjero y este personal, estarán exentos, para los efectos de esos contratos, del cumplimiento de las leyes de previsión que rijan para los trabajadores, no estando obligados, en consecuencia, a efectuar imposiciones de ninguna naturaleza en organismos de previsión chilenos, siempre que se reúnan las siguientes condiciones:

a) Que el trabajador se encuentre afiliado a un régimen de previsión o de seguridad social fuera de Chile, cualquiera sea su naturaleza jurídica, que le otorgue prestaciones, a lo menos, en casos de enfermedad, invalidez, vejez y muerte, y 
b) Que en el contrato de trabajo respectivo el trabajador exprese su voluntad de mantener la afiliación referida.

La exención que establece el inciso anterior no comprenderá los riesgos de accidentes del trabajo y enfermedades profesionales previstos en la Ley $N^{\circ} 16.744^{\prime \prime}$.

La exención de cotizar dice relación con algunos riesgos y regímenes específicos. Así se debe igualmente cotizar para la ley de accidentes del trabajo y enfermedades profesionales. Además, como ha señalado la Dirección del Trabajo $^{1}$, los trabajadores extranjeros se encuentran obligados a efectuar cotizaciones por concepto de seguro de cesantía. Esto debido a que la exención del artículo $1^{\circ}$ de la Ley $N^{\circ} 18.156$ sólo ópera respecto de los riesgos enfermedad, vejez, invalidez y muerte. Tiene, entonces, un alcance material limitado.

Esta ley permite al Técnico Extranjero, en su artículo $7^{\circ}$, la posibilidad, de recuperar lo cotizado en una administradora de fondos de pensiones chilena, siempre que se cumplan los requisitos del artículo $1^{\circ}$ y realizando la solicitud correspondiente. En el caso de este comentario el trabajador realizó la solicitud pero la AFP no quiso devolver los fondos.

A esta altura debemos hacer notar los posibles efectos perversos de esta normativa sobre protección de riesgos sociales de los trabajadores. Si no están afiliados en Chile su cobertura se verá comprometida sobre todo en relación a la enfermedad ${ }^{2}$. ¿Qué pasa si el trabajador se enferma en Chile? ¿Cómo cubrirá la licencia médica o los gastos hospitalarios? Estas son cuestiones que deben ser debatidas y reguladas en un futuro cercano.

En cuanto a la vejez y a la posibilidad que nos presenta esta reglamentación, debemos señalar que se trata de un caso muy particular. En general, para poder tener derecho a pensión de vejez las legislaciones establecen ciertas condiciones, de esta manera las pensiones se entregan generalmente con base en períodos de calificación, de actividad o de residencia. Además, la afiliación al sistema nacional de seguridad social puede ser un requisito para la obtención de beneficios, como, por ejemplo, protección en caso de enfermedad o en caso de accidente de trabajo. Es decir, para obtener prestaciones se debe cumplir con ciertas condiciones. En general, estas condiciones son cumplidas por el trabajador migrante en situación migratoria regular y que pertenece el sector formal ${ }^{3}$, ya que éste,

\footnotetext{
${ }^{1}$ Dictamen Ord. № 1.539/017 de 28 de marzo de 2012.

${ }^{2}$ Arellano Ortiz, Pablo Andrés, "Trabajadores migrantes y seguridad social: aproximación nacional e internacional a los mecanismos de protección que otorgan continuidad a sus prestaciones, II Parte", Revista Chilena de Derecho de Trabajo y de la Seguridad Social, Departamento de Derecho de Trabajo y de la Seguridad Social, Facultad de Derecho, Universidad de Chile, vol. 4, № 7, 2013, pp. 119-133

${ }^{3}$ Hirose, Kenichi; NiKac, Milos y Tamagno, Edward, Social security for migrant workers. A right-based approach, International Labour Organization, Decent Work Technical Support Team, and Country Office for Central and Eastern Europe-Budapest, ILO, 2011, p. 2.
} 
al poseer una situación migratoria regular, se encuentra en general afiliado a la seguridad social del país anfitrión.

Pero, ¿qué sucede con los derechos en curso de adquisición y con las cotizaciones aportadas en otros países donde el migrante ha trabajado? Para poder resolver estas cuestiones, la seguridad social ha recurrido a un tipo de tratados internacionales que permiten a los Estados signatarios otorgar al trabajador migrante una cierta continuidad en la adquisición de sus prestaciones y en la cobertura misma. Sin embargo, la complejidad de la migración ha implicado que cada vez más trabajadores migrantes corran el riesgo de perder sus derechos a cobertura de seguridad social ${ }^{4}, y$, por otro lado, la existencia de un sector de la economía informal de proporciones importantes, ha dificultado la entrega de mecanismos con coberturas suficientes.

La regulación de la situación de los migrantes ha tomado diversas formas, en la búsqueda de establecer un mecanismo que permita la continuidad de prestaciones para los trabajadores migrantes y sus familias. Para ello se han adoptado diferentes medidas a nivel internacional. Existen normas internacionales relativas a derechos humanos, normas internacionales de la Organización Internacional del Trabajo (OIT), y también regulaciones establecidas por los propios países a nivel unilateral, bilateral y multilateral.

La Convención internacional sobre la protección de los derechos de todos los trabajadores migratorios y de sus familiares, adoptada por la Asamblea General en su Resolución № 45/158, de 18 de diciembre de 1990, constituye el principal instrumento en materia de derechos humanos para los trabajadores migrantes y sus familias. Esta convención fue firmada por nuestro país el 24 de septiembre de 1993 y ratificada el 21 marzo de 2005. Este instrumento contiene disposiciones específicas relativas a las prestaciones de seguridad social para los migrantes y sus familias, a saber, en el artículo 27 se señala que:

"Los trabajadores migratorios y sus familiares gozarán en el Estado de empleo, con respecto a la seguridad social, del mismo trato que los nacionales en la medida en que cumplan los requisitos previstos en la legislación aplicable de ese Estado o en los tratados bilaterales y multilaterales aplicables. Las autoridades competentes del Estado de origen y del Estado de empleo podrán tomar en cualquier momento las disposiciones necesarias para determinar las modalidades de aplicación de esta norma".

El mismo artículo agrega:

"Cuando la legislación aplicable no permita que los trabajadores migratorios o sus familiares gocen de alguna prestación, el Estado de que se trate, sobre la base del trato otorgado a los nacionales que estuvieren en situación similar, considerará la posibilidad de reembolsarles el monto de las contribuciones que hubieren aportado en relación con esas prestaciones".

${ }^{4}$ Hirose, Nikac y Tamagno, cit. nota n. 3, p. 2. 
Dicho de otra manera: los sistemas de seguridad social eventualmente pueden devolver al trabajador migrante los montos aportados, pero ello debe ser efectuado sobre la base de igualdad de trato con el nacional. Si al nacional se le permite, al extranjero también. Cabe señalar que en nuestra legislación al nacional no se le permite retirar los fondos antes de su jubilación. Estaríamos entonces ante una medida en favor del migrante. Medida que en un principio compartimos.

Por otro lado, existen otros instrumentos internacionales, entre los cuales encontramos la Convención internacional sobre la protección de los derechos de todos los trabajadores migratorios y de sus familiares, el Convenio sobre los trabajadores migrantes (revisado), 1949 (núm. 97) y el Convenio sobre los trabajadores migrantes (disposiciones complementarias), 1975 (núm. 143) de la OIT. Además, existen los acuerdos bilaterales, que tienen por objeto entregar una continuidad en la cobertura al conectar dos legislaciones. En este proceso, en general, no se intercambian dineros, sino que más bien se reconocen periodos de afiliación o de cotización que permitan al migrante poder cumplir con los requisitos necesarios para tener derecho a una prestación.

Dentro de la evolución de los instrumentos internacionales encontramos instrumentos que se presentan como una solución a largo plazo a la cobertura de seguridad social para migrantes, como, por ejemplo, el Convenio Multilateral Iberoamericano de Seguridad Social (CMISS), vigente en nuestro país desde el 1 de septiembre de 2011.

Estas últimas normas internacionales, de diversas formas, lo que pretenden es entregarle continuidad a las prestaciones del trabajador migrante. Que lo cotizado en un país sirva para optar a una prestación en otro país. Es así como nuestro país ha firmado una seria de acuerdos bilaterales de seguridad social y además el CMISS. La gran parte de estos instrumentos dicen relación con las prestaciones de vejez. Lamentablemente, no hay una aproximación completa y coherente a todos los riesgos sociales. Consideramos, eso sí, al CMISS como el primer paso hacia un entramado más completo al respecto ${ }^{5}$.

Volviendo al caso de esta sentencia, en principio nuestra legislación no está errada al permitir recuperar los fondos al trabajador migrante. Sin embargo, debemos, a la luz de lo expuesto, realizar algunas observaciones:

Primero, el trabajador migrante recupera los fondos en virtud del derecho de propiedad. Sin embargo, parece existir un derecho de propiedad distinto para el trabajador migrante. Esto porque el nacional no puede ejercer este mismo derecho para recuperar sus fondos acumulados.

Segundo, la entrega de fondos puede hacerse de acuerdo a la normativa internacional, pero esta exige que también se le permita hacer lo mismo al trabajador nacional.

${ }^{5}$ Arellano Ortiz, cit. nota n. 2. 
Tercero, lo más grave es que no se estaría cumpliendo con el objetivo de que el trabajador migrante logre una continuidad en las prestaciones. El trabajador termina sus labores en el país, retira sus cotizaciones, pero estas no le servirán para obtener derecho a una pensión.

Nuestro país posee una inmigración creciente y a la vez una emigración que comienza a ser importante. La situación descrita en este caso se hará cada vez más frecuente. Es así, entonces, que encontraremos casos en que los trabajadores migrantes se vean expuestos a quedar sin una pensión de vejez. Nuestra legislación deberá en los próximos años buscar el mecanismo que permita a todo trabajador, independiente de su nacionalidad, poder adquirir la posibilidad de obtener un derecho a pensión.

Santiago, veinticuatro de junio de dos mil trece.

\section{Vistos y teniendo presente:}

$\left.1^{\circ}\right)$ Que a fojas 7 comparece don Juan Agustín Arturo, representado por doña Antonia Illanes Riquelme, quien dedujo acción de protección en contra de la AFP Habitat S.A., representada por don Cristian Rodríguez Allendes, por el acto que estima arbitrario e ilegal, consistente en que dicha Administradora le negó la entrega de las cotizaciones previsionales que tiene en su poder y que son de su propiedad. Sostiene el recurrente que es de nacionalidad argentina y de profesión ingeniero, que trabajó desde el 26 de noviembre de 1992 al 11 de mayo de 1994, en la Empresa Aceros Chile, C.C.S.A., en Chile. Al momento de suscribir el contrato de trabajo se encontraba afiliado al régimen previsional de su país de origen, manifestando expresamente en el contrato de trabajo su intención de mantener la afiliación con el régimen previsional argentino. Durante sus labores en esa empresa, enteró las cotizaciones previsionales en la recurrida. Al terminar su contrato, volvió a trabajar a Argentina. Desde 2008 comenzó a realizar las gestiones para la devolución del dinero retenido y que es de su patrimonio personal, lo que negó la AFP Habitat aludiendo a que no se adjuntó el contrato de trabajo. Si bien no tenía copia del contrato, tenía copia del finiquito, que acompaña al recurso, en el que se dejó constancia de la existencia de sus aportes previsionales y de salud en su país de origen, los que mantuvo para los efectos legales. Reclamada la intervención de la Superintendencia de Pensiones, su respuesta fue negativa. La actuación de la recurrida afecta la garantía constitucional que se establece a su favor en el artículo 19 № 24 de la Carta Fundamental e importa una disminución concreta y efectiva a su patrimonio.

$2^{\boldsymbol{o}}$ ) A fs. 39 y siguientes rola el informe de la Administradora de Fondos de pensiones Habitat S.A., afirmando primeramente que en su parecer, este recurso es improcedente en atención a su naturaleza cautelar ante un acto u omisión arbitrarios. En la especie, no ha realizado ningún acto arbitrario o ilegal que haya impedido el ejercicio de derechos constitucionales, ha dado cumplimiento a la 
ley y la normativa aplicable para la determinación de los requisitos establecidos en la Ley № 18.156, sobre Devolución de Fondos de Técnicos Extranjeros. El recurrente tiene derecho a ejercer su defensa en los procesos ordinarios y no corresponde que esta materia sea resuelta mediante esta vía. En concreto, mediante la norma de la letra c) del artículo 420 del Código del Trabajo, que así lo establece.

En cuanto a los hechos, afirma que el señor Arturo mantiene en su cuenta individual de cotizaciones obligatorias, un saldo de 258,64 cuotas, equivalentes al 15 de mayo de 2013 a la suma de $\$ 6.052 .039$ y no registra solicitud del recurrente para obtener la devolución de ellas. La Ley $N^{\circ} 18.156$, modificada por la Ley $N^{\circ} 18.726$, contempla en el artículo $7^{\circ}$ la situación de trabajadores extranjeros que registren cotizaciones en una Administradora de Fondos de Pensiones, los que podrán solicitar la devolución de los fondos. En lo particular, se requiere que el contrato de trabajo contenga una clausula relativa a la mantención de la afiliación por parte del trabajador, a un régimen de previsión o seguridad fuera de Chile. Este requisito es de carácter esencial. La Ley № 18.156 es una norma especial y de excepción, que debe interpretarse restrictivamente. La Superintendencia de Pensiones, en tanto fija la interpretación de la legislación y reglamentación del sistema, es de carácter obligatorio para las Administradoras de Fondos de Pensiones y se ha pronunciado sostenida y reiteradamente que se debe dar cumplimiento a los requisitos de la Ley $N^{\circ} 18.156$ para la devolución de los fondos previsionales.

Sostiene que no ha cometido acto arbitrario o ilegal, y que en todo caso, no cuenta con registros sobre la solicitud alguna del recurrente para la devolución de sus fondos.

$3^{\circ}$ ) La actuación de la recurrida, la Administradora de Fondos de Pensiones Habitat S.A. consistió en negarse a devolver los fondos previsionales que había enterado a su favor, por el tiempo trabajado en Chile, no obstante haber expresado su voluntad de mantener su afiliación al régimen previsional de su país de origen, Argentina, atendido a que no se acompañó copia del contrato de trabajo en que conste la expresión de voluntad del trabajador extranjero, afectándose de tal manera el derecho de propiedad de consagración constitucional y, por ende, amparable por esta acción cautelar.

La inexistencia escrita del contrato de trabajo no fue cuestión que las partes controvirtieron en el recurso. No obstante, debe dejarse sentado que aquel instrumento tiene una finalidad de acreditación de la existencia de un contrato de trabajo con un técnico extranjero y de la voluntad de éste de mantener el sistema previsional del que gozaba en su país de origen.

Ante ello, la existencia del finiquito que rola a fs. 2, suscrito entre la empresa Aceros Chile C.C.S.A. y el recurrente, en que dejó constancia del tiempo servido por éste para aquella, 26 de noviembre de 1992 y hasta el 4 de mayo de 1994, 
así como la voluntad del trabajador de mantener sus aportes previsionales y de salud en su país natal, los que mantiene para los efectos legales, son prueba suficiente y apta para acreditar con fuerza vinculatoria para la recurrida, los requisitos y presupuestos que exige el artículo $1^{\circ}$ de la Ley $N^{\circ} 18.156$, que permite expresamente la restitución de los fondos previsionales a los trabajadores extranjeros.

El texto legal anterior impone la obligación de restitución de aquellos fondos, y no se alegó ni se vislumbró en los alegatos en estrados, la existencia de alguna causal de retención de los mismos.

$4^{\circ}$ ) Aquel actuar de la recurrida afecta efectivamente la garantía invocada, esto es, el derecho de propiedad sobre aquellos fondos, al retenerlos en su poder y administrarlos según su parecer, cuando existe el derecho del trabajador extranjero de obtener su restitución por integrar su patrimonio.

Por lo anterior, el presente recurso de protección debe ser acogido.

Por estas consideraciones, y de acuerdo, también con lo preceptuado en los artículos 19 y 20 de la Constitución Política de la República, y en el Auto Acordado de la Excma. Corte Suprema sobre Tramitación y Fallo del Recurso de Protección, se acoge el recurso de protección deducido a fojas 7 , con costas, ordenándose que la recurrida haga devolución de los fondos previsionales que mantiene en su poder y que son de propiedad de don Juan Agustín Arturo, según valor de la cuota al día de su pago efectivo.

Regístrese, comuníquese y archívese en su oportunidad.

Redactada por el Ministro (S) señor Carrillo González.

No Protección 45645-2012.

Pronunciada por la Segunda Sala de esta Iltma. Corte de Apelaciones de Santiago, presidida por el Ministro, presidida por el Ministro señor Miguel Vázquez Plaza e integrada por el Ministro (S) señor Carlos Carrillo González y por el Abogado Integrante señor Ángel Cruchaga Gandarillas.

Autorizado por el (la) ministro de fe de esta Corte de Apelaciones.

En Santiago, a veinticuatro de junio de dos mil trece, notifiqué en secretaría por el estado diario la resolución precedente. 